\title{
AN EXISTENCE THEOREM FOR MODEL EQUATIONS RESULTING FROM KINETIC THEORIES OF POLYMER SOLUTIONS
}

By

Michael Renardy

IMA Preprint Series \# 588

October 1989 


\section{Introduction}

General existence theorems for the partial differential equations of continuum mechanics are relatively recent, even for the case of elasticity. The initial value problem for compressible elastic materials occupying all of space was solved in [6], and the Dirichlet initial-boundary value problem is treated in [7], [2] and [3]. Existence results for incompressible materials were obtained in [4] and [11] for the initial value problem on all of space and in [5] for the Dirichlet problem.

Viscoelastic fluids such as polymers are characterized by a constitutive relation which gives the stress as a functional of the deformation history. Most popular models fall into two categories: integral and differential models. Integral models give the stress by an integral expression involving the history of the deformation gradient; differential models give the stress as the solution of a differential equation which involves the stress and velocity gradient. There is, however, a class of models motivated by considerations of kinetic theory, which can in general not be represented in either integral or differential form. Instead, such models require the solution of a diffusion equation in order to determine the stress [1]. Because of the practical impossibility - except in special cases - of solving this diffusion equation, such models have only had limited applications in solving flow problems. For the case of dumbbell models, however, computers may soon have the capacity to obtain solutions, at least in two-dimensional flow problems.

For materials with instantaneous elasticity, the terms of leading differential order in the equations of motion are like those for elasticity. A natural approach to a mathematical existence theory is therefore to regard the problem as essentially "elastic" and to treat the memory as a perturbation. This has been successfully carried out for integral models, see e.g. Chapter III of [8], and also for differential models [10]. In this paper, we shall discuss the models from kinetic theory in a similar fashion. We shall in part be able to rely on the results of $[10]$, but the solution of the diffusion equation raises new issues.

The equations of motion for an incompressible fluid are

$$
\begin{gathered}
\rho\left(\frac{\partial \mathbf{v}}{\partial t}+(\mathbf{v} \cdot \nabla) \mathbf{v}\right)=\operatorname{div} \mathbf{T}-\nabla p+\mathbf{f} \\
\operatorname{div} \mathbf{v}=0 .
\end{gathered}
$$

Here $\mathbf{v}$ denotes the velocity, $p$ the pressure, $\mathbf{T}$ the extra stress tensor, $\rho$ the density (assumed constant), and $\mathbf{f}$ is a given body force. We investigate molecular models which are based on treating polymer molecules as dumbbells consisting of two beads connected by a spring and floating in a Newtonian solvent. We shall assume that the solvent contribution to the stress is small relative to the polymer contribution and can be neglected; if this is not the case, the equations of motion can be treated as a perturbation of the Stokes equations, and existence results can be obtained along the lines of [9]. Let $\mathbf{R}$ denote the vector between the two ends of the dumbbell, and let $\psi(\mathbf{R}, \mathbf{x}, t)$ denote the probability density for dumbbells in $\mathbf{R}$-space (this probability density depends on the point $\mathbf{x}$ in space and the time $t$ ). The spring force is given by $\mathbf{F}(\mathbf{R})=-\gamma\left(|\mathbf{R}|^{2}\right) \mathbf{R}$, and the stress is given by

$$
T_{i j}(\mathbf{x}, t)=-n \int R_{i} F_{j}(\mathbf{R}) \psi(\mathbf{R}, \mathbf{x}, t) d \mathbf{R} .
$$


Here $n$ is the number density of dumbbells and the integral extends over all possible values of $\mathbf{R}$. We shall consider infinitely extensible as well as finitely extensible dumbbells. For infinitely extensible dumbbells, $\mathbf{R}$ can be any vector in $\mathbb{R}^{3}$. For finitely extensible dumbbells, the potential associated with $\mathbf{F}(\mathbf{R})$ becomes infinite at a finite value $R_{0}$ of $|\mathbf{R}|$, and the integral in (2) extends only over the ball $|\mathbf{R}| \leq R_{0}$. The probability density $\psi(\mathbf{R}, \mathbf{x}, t)$ obeys the diffusion equation

$$
\left(\frac{\partial}{\partial t}+\left(\mathbf{v} \cdot \nabla_{\mathbf{x}}\right)\right) \psi=\alpha \Delta_{\mathbf{R}} \psi+\operatorname{div}_{\mathbf{R}}\left(-\left(\nabla_{\mathbf{x}} \mathbf{v}\right) \cdot \mathbf{R} \psi-\beta \mathbf{F}(\mathbf{R}) \psi\right) .
$$

Here we have used subscripts $\mathbf{R}$ and $\mathbf{x}$ to clarify with respect to which variables derivatives are being taken. Our convention for the gradient of a vector is that the first index refers to the component of the vector and the second index to the direction of differentiation. The quantities $\alpha$ and $\beta$ are positive constants. The reader is referred to [1] for the derivation of (2) and (3) and for a discussion of possible generalizations.

We seek solutions of (1)-(3) for $\mathrm{x} \in \Omega, t>0$, where $\Omega$ is a bounded domain in $\mathbb{R}^{3}$ with smooth boundary. We impose Dirichlet boundary conditions,

$$
\mathbf{v}(\mathbf{x}, t)=\mathbf{0}, \mathbf{x} \in \partial \Omega, t \geq 0,
$$

and initial conditions,

$$
\mathbf{v}(\mathbf{x}, 0)=\mathbf{v}_{0}(\mathbf{x}), \psi(\mathbf{R}, \mathbf{x}, 0)=\psi_{0}(\mathbf{R}, \mathbf{x}) .
$$

Concerning the spring force, we shall make one of the following two assumptions:

(F) The function $\gamma$ is $C^{\infty}$-smooth from $[0, \infty)$ to $(0, \infty), \gamma^{\prime} \geq 0$, and there exist numbers $\sigma \geq 0$ and $k>0$ such that $\lim _{|\mathbf{R}| \rightarrow \infty} \gamma\left(|\mathbf{R}|^{2}\right) /|\mathbf{R}|^{\sigma}=k$. Moreover, $\lim \sup _{|\mathbf{R}| \rightarrow \infty} \gamma^{\prime}\left(|\mathbf{R}|^{2}\right) /|\mathbf{R}|^{\sigma-2}$ is finite and higher derivatives of $\gamma$ have at most polynomial growth as $|\mathbf{R}| \rightarrow \infty$.

$\left(\mathrm{F}^{\prime}\right)$ For some $R_{0}>0$, the function $\gamma$ is $C^{\infty}$-smooth from $\left[0, R_{0}^{2}\right)$ to $(0, \infty), \gamma^{\prime} \geq 0$, and there exist numbers $\sigma>1$ and $k>0$ such that $\lim _{|\mathbf{R}| \rightarrow R_{0}} \gamma\left(|\mathbf{R}|^{2}\right)\left(R_{0}-|\mathbf{R}|\right)^{\sigma}=k$. Moreover, $\lim \sup _{|\mathbf{R}| \rightarrow R_{0}} \gamma^{\prime}\left(|\mathbf{R}|^{2}\right)\left(R_{0}-|\mathbf{R}|\right)^{\sigma+1}$ is finite and higher derivatives of $\gamma$ grow at must like powers of $\left(R_{0}-|\mathbf{R}|\right)^{-1}$ as $|\mathbf{R}| \rightarrow R_{0}$.

In the first case, the dumbbell is infinitely extensible, in the second case it is finitely extensible. The assumption $\gamma^{\prime} \geq 0$ means that the spring gets stiffer as the dumbbell is extended; this assumption is usually made in molecular models. The analysis that follows can be modified to allow faster growth of $\gamma$ as $|\mathbf{R}| \rightarrow \infty$ or, respectively, $|\mathbf{R}| \rightarrow R_{0}$, but slower growth would pose difficulties. In particular, we have to exclude $\sigma=1$ in $\left(\mathrm{F}^{\prime}\right)$. This case corresponds to the most popular model of finitely extensible dumbbells (see [1]). There is, however, no specific reason to assume $\sigma=1$ rather than a larger value of $\sigma$.

The goal of this paper is an existence and uniqueness theorem for solutions of the problem defined by (1)-(5). The paper is organized as follows. In Section 2, we define an iteration scheme which is used to construct the solution. The scheme alternates between solving an equation of the same type as encountered in incompressible elasticity and solving a linear diffusion equation such as (3). In Section 3 we define the function spaces used to carry out the analysis and state a precise theorem. The proof is given in Section 4. 


\section{Iterative construction of solution}

We apply the operation $\frac{\partial}{\partial t}+(\mathbf{v} \cdot \nabla)+(\nabla \mathbf{v})^{T}$ to the equation of motion (1). The resulting equation, written in components, reads

$$
\begin{gathered}
\rho\left[\frac{\partial}{\partial t}+(\mathbf{v} \cdot \nabla)\right]^{2} v_{i}=\frac{\partial}{\partial x_{j}}\left[\left(\frac{\partial}{\partial t}+(\mathbf{v} \cdot \nabla)\right) T_{i j}\right]-\frac{\partial q}{\partial x_{i}} \\
-\frac{\partial v_{k}}{\partial x_{j}} \frac{\partial T_{i j}}{\partial x_{k}}+\frac{\partial v_{k}}{\partial x_{i}} \frac{\partial T_{k j}}{\partial x_{j}}-\rho \frac{\partial v_{j}}{\partial x_{i}}\left[\frac{\partial}{\partial t}+(\mathbf{v} \cdot \nabla)\right] v_{j} \\
+\left[\frac{\partial}{\partial t}+(\mathbf{v} \cdot \nabla)\right] f_{i}+\frac{\partial v_{j}}{\partial x_{i}} f_{j}
\end{gathered}
$$

where $q=\frac{\partial p}{\partial t}+(\mathbf{v} \cdot \nabla) p$. Using equation (2), we find that

$$
\left[\frac{\partial}{\partial t}+(\mathbf{v} \cdot \nabla)\right] T_{i j}=-n \int R_{i} F_{j}(\mathbf{R})\left[\frac{\partial}{\partial t}+\left(\mathbf{v} \cdot \nabla_{\mathbf{x}}\right)\right] \psi(\mathbf{R}, \mathbf{x}, t) d \mathbf{R}
$$

and using equation (3), we find further

$$
\begin{gathered}
{\left[\frac{\partial}{\partial t}+(\mathbf{v} \cdot \nabla)\right] T_{i j}=-n \int R_{i} F_{j}(\mathbf{R})\left[\alpha \Delta_{\mathbf{R}} \psi+\operatorname{div}_{\mathbf{R}}\left(-\left(\nabla_{\mathbf{x}} \mathbf{v}\right) \cdot \mathbf{R} \psi-\beta \mathbf{F}(\mathbf{R}) \psi\right)\right] d \mathbf{R}} \\
=-n \alpha \int \Delta_{\mathbf{R}}\left(R_{i} F_{j}(\mathbf{R})\right) \psi d \mathbf{R}-n \beta \int \frac{\partial}{\partial R_{k}}\left(R_{i} F_{j}(\mathbf{R})\right) F_{k}(\mathbf{R}) \psi d \mathbf{R} \\
-n \int \frac{\partial}{\partial R_{k}}\left(R_{i} F_{j}(\mathbf{R})\right) \frac{\partial v_{k}}{\partial x_{l}} R_{l} \psi d \mathbf{R} .
\end{gathered}
$$

Next we note that

$$
\frac{\partial}{\partial R_{k}}\left(R_{i} F_{j}(\mathbf{R})\right)=-\frac{\partial}{\partial R_{k}}\left(R_{i} R_{j} \gamma\left(|\mathbf{R}|^{2}\right)\right)=-\gamma\left(|\mathbf{R}|^{2}\right)\left(R_{j} \delta_{i k}+R_{i} \delta_{j k}\right)-2 R_{i} R_{j} R_{k} \gamma^{\prime}\left(|\mathbf{R}|^{2}\right) .
$$

By combining (8) and (9), we find

$$
\begin{gathered}
\frac{\partial}{\partial x_{j}}\left[\left(\frac{\partial}{\partial t}+\mathbf{v} \cdot \nabla\right) T_{i j}\right]= \\
-n \alpha \int \Delta_{\mathbf{R}}\left(R_{i} F_{j}(\mathbf{R})\right) \frac{\partial \psi}{\partial x_{j} \partial x_{l}} \int\left(\gamma\left(|\mathbf{R}|^{2}\right) R_{j} R_{l} \delta_{i k}+2 \gamma^{\prime}\left(|\mathbf{R}|^{2}\right) R_{i} R_{j} R_{k} R_{l}\right) \psi d \mathbf{R} \\
-n \frac{\partial v_{k}}{\partial x_{l}} \int \frac{\partial}{\partial R_{k}}\left(R_{i} F_{j}(\mathbf{R})\right) F_{k}(\mathbf{R}) \frac{\partial \psi}{\partial x_{j}} d \mathbf{R} \\
-(\mathbf{R})) R_{l} \frac{\partial \psi}{\partial x_{j}} d \mathbf{R} .
\end{gathered}
$$

By inserting (10) into (6), we finally obtain

$$
\rho\left[\frac{\partial}{\partial t}+(\mathbf{v} \cdot \nabla)\right]^{2} v_{i}=n \frac{\partial^{2} v_{k}}{\partial x_{j} \partial x_{l}} \int\left(\gamma\left(|\mathbf{R}|^{2}\right) R_{j} R_{l} \delta_{i k}+2 \gamma^{\prime}\left(|\mathbf{R}|^{2}\right) R_{i} R_{j} R_{k} R_{l}\right) \psi d \mathbf{R}-\frac{\partial q}{\partial x_{i}}
$$




$$
\begin{gathered}
-n \alpha \int \Delta_{\mathbf{R}}\left(R_{i} F_{j}(\mathbf{R})\right) \frac{\partial \psi}{\partial x_{j}} d \mathbf{R}-n \beta \int \frac{\partial}{\partial R_{k}}\left(R_{i} F_{j}(\mathbf{R})\right) F_{k}(\mathbf{R}) \frac{\partial \psi}{\partial x_{j}} d \mathbf{R} \\
-n \frac{\partial v_{k}}{\partial x_{l}} \int \frac{\partial}{\partial R_{k}}\left(R_{i} F_{j}(\mathbf{R})\right) R_{l} \frac{\partial \psi}{\partial x_{j}} d \mathbf{R} \\
-\frac{\partial v_{k}}{\partial x_{j}} \frac{\partial T_{i j}}{\partial x_{k}}+\frac{\partial v_{k}}{\partial x_{i}} \frac{\partial T_{k j}}{\partial x_{j}}-\rho \frac{\partial v_{j}}{\partial x_{i}}\left[\frac{\partial}{\partial t}+(\mathbf{v} \cdot \nabla)\right] v_{j} \\
+\left[\frac{\partial}{\partial t}+(\mathbf{v} \cdot \nabla)\right] f_{i}+\frac{\partial v_{j}}{\partial x_{i}} f_{j} .
\end{gathered}
$$

The iteration is based on equations (3) and (11). Given an iterate $\mathbf{v}^{m}$, we determine $\psi^{m}$ by solving equation (3),

$$
\begin{gathered}
\left(\frac{\partial}{\partial t}+\left(\mathbf{v}^{m} \cdot \nabla_{\mathbf{x}}\right)\right) \psi^{m}=\alpha \Delta_{\mathbf{R}} \psi^{m}+\operatorname{div}_{\mathbf{R}}\left(-\left(\nabla_{\mathbf{x}} \mathbf{v}^{m}\right) \cdot \mathbf{R} \psi^{m}-\beta \mathbf{F}(\mathbf{R}) \psi^{m}\right) \\
\psi^{m}(\mathbf{R}, \mathbf{x}, 0)=\psi_{0}(\mathbf{R}, \mathbf{x}) .
\end{gathered}
$$

We define $\mathbf{T}^{m}$ in terms of $\psi^{m}$ by equation (2) and then determine a new velocity field $\mathbf{v}^{m+1}$ by solving the problem

$$
\begin{gathered}
\rho\left[\frac{\partial}{\partial t}+\left(\mathbf{v}^{m} \cdot \nabla\right)\right]^{2} v_{i}^{m+1} \\
=n \frac{\partial^{2} v_{k}^{m+1}}{\partial x_{j} \partial x_{l}} \int\left(\gamma\left(|\mathbf{R}|^{2}\right) R_{j} R_{l} \delta_{i k}+2 \gamma^{\prime}\left(|\mathbf{R}|^{2}\right) R_{i} R_{j} R_{k} R_{l}\right) \psi^{m} d \mathbf{R}-\frac{\partial q^{m+1}}{\partial x_{i}} \\
-n \alpha \int \Delta_{\mathbf{R}}\left(R_{i} F_{j}(\mathbf{R})\right) \frac{\partial \psi^{m}}{\partial x_{j}} d \mathbf{R}-n \beta \int \frac{\partial}{\partial R_{k}}\left(R_{i} F_{j}(\mathbf{R})\right) F_{k}(\mathbf{R}) \frac{\partial \psi^{m}}{\partial x_{j}} d \mathbf{R} \\
-n \frac{\partial v_{k}^{m}}{\partial x_{l}} \int \frac{\partial}{\partial R_{k}}\left(R_{i} F_{j}(\mathbf{R})\right) R_{l} \frac{\partial \psi^{m}}{\partial x_{j}} d \mathbf{R} \\
-\frac{\partial v_{k}^{m}}{\partial x_{j}} \frac{\partial T_{i j}^{m}}{\partial x_{k}}+\frac{\partial v_{k}^{m}}{\partial x_{i}} \frac{\partial T_{k j}^{m}}{\partial x_{j}}-\rho \frac{\partial v_{j}^{m}}{\partial x_{i}}\left[\frac{\partial}{\partial t}+\left(\mathbf{v}^{m} \cdot \nabla\right)\right] v_{j}^{m} \\
+\left[\frac{\partial}{\partial t}+\left(\mathbf{v}^{m} \cdot \nabla\right)\right] f_{i}+\frac{\partial v_{j}^{m}}{\partial x_{i}} f_{j}, \\
\operatorname{div} \mathbf{v}^{m+1}=0 \\
\mathbf{v}^{m+1}=\mathbf{0}, x \in \partial \Omega, t \geq 0 . \\
\mathbf{v}^{m+1}(\mathbf{x}, 0)=\mathbf{v}_{0}(\mathbf{x}) .
\end{gathered}
$$




\section{Definition of function spaces and statement of results}

Since $\psi$ has the meaning of a probability density, $L^{1}$-spaces are natural for the $\mathbf{R}$ dependence. However, the definition of $\mathbf{T}$ in equation (2) involves integrals of $\psi$ against increasing functions of $\mathbf{R}$, and we shall therefore use weighted $L^{1}$-spaces. We define

$$
X_{n}=\left\{\psi: \mathbb{R}^{3} \rightarrow \mathbb{R}\left|\int\left(1+|\mathbf{R}|^{n}\right)\right| \psi(\mathbf{R}) \mid d \mathbf{R}<\infty\right\},
$$

in the case of infinitely extensible dumbbells, and

$$
X_{n}=\left\{\psi: B \rightarrow \mathbb{R}\left|\int\left(R_{0}-|\mathbf{R}|\right)^{-n}\right| \psi(\mathbf{R}) \mid d \mathbf{R}<\infty\right\},
$$

in the case of finitely extensible dummbells. Here $B$ denotes the ball of radius $R_{0}$. Moreover, we let $X_{n, k}$ be the space of all $\psi$ whose derivatives up to order $k$ lie in $X_{n}$. Finally, let

$$
X_{k}=\bigcap_{n=0}^{\infty} X_{n, k}
$$

with the natural topology of a Fréchet space.

We make the following smoothness assumptions:

(S1) The domain $\Omega \subset \mathbb{R}^{3}$ is bounded and $\partial \Omega$ is of class $C^{5}$.

(S2) $\mathbf{v}_{0} \in H^{4}(\Omega)$.

(S3) $\psi_{0} \in \bigcap_{k=0}^{4} H^{k}\left(\Omega ; X_{8-2 k}\right)$, where $H^{k}\left(\Omega ; X_{l}\right)$ stands for $\bigcap_{n=0}^{\infty} H^{k}\left(\Omega ; X_{n, l}\right)$. Moreover, $\psi_{0} \geq 0$ and $\int \psi_{0}(\mathbf{R}, \mathbf{x}) d \mathbf{R}=1$ for every $\mathbf{x} \in \Omega$.

(S4) For some $T>0$, we have $\mathbf{f} \in \bigcap_{k=0}^{4} W^{k, 1}\left([0, T] ; H^{4-k}(\Omega)\right)$.

In addition, we need compatibility conditions between the initial data and the incompressibility and boundary conditions. Note that by applying the Hodge projection operator to equation (1), we can obtain an initial value for $\partial \mathbf{v} / \partial t$, and, after differentiating (1) with respect to $t$, we can obtain initial values of higher time derivatives in an analogous fashion. We shall denote the initial value of $\partial^{i} \mathbf{v} / \partial t^{i}$ by $\mathbf{v}_{i}$. These initial values for time derivatives of $\mathbf{v}$ satisfy the incompressibility condition because of their construction, but we still need to require that they satisfy the boundary conditions. We assume the following compatibility conditions:

(C1) $\operatorname{div} \mathbf{v}_{\mathbf{0}}=0$ and $\mathbf{v}_{\mathbf{0}}=\mathbf{0}$ on $\partial \Omega$.

(C2) $\mathbf{v}_{1}, \mathbf{v}_{2}$ and $\mathbf{v}_{3}$ vanish on $\partial \Omega$.

The goal of the paper is the following result.

\section{Theorem:}

Assume that (S1)-(S4), (C1), (C2) and (F) or, respectively, $\left(\mathrm{F}^{\prime}\right)$ hold. Then there is a $T^{\prime} \in(0, T]$ such that the problem (1)-(5) has a unique solution with the regularity

$$
\mathbf{v} \in \bigcap_{k=0}^{4} C^{k}\left(\left[0, T^{\prime}\right] ; H^{4-k}(\Omega)\right) ; \mathbf{T} \in \bigcap_{k=0}^{3} C^{k}\left(\left[0, T^{\prime}\right] ; H^{3-k}(\Omega)\right) ;
$$




$$
\psi \in \bigcap_{k=0}^{3} \bigcap_{l=0}^{3-k} C^{k}\left(\left[0, T^{\prime}\right] ; H^{l}\left(\Omega ; X_{8-2 k-2 l}\right)\right)
$$

The proof will be based on showing that the mapping $\Sigma: \mathbf{v}^{m} \rightarrow \mathbf{v}^{m+1}$ defined by (12) and (13) is a contraction in an appropriate complete space of functions. This space of functions, denoted by $Z\left(M, T^{\prime}\right)$, is defined as the set of all functions $\mathbf{v}: \Omega \times\left[0, T^{\prime}\right] \rightarrow \mathbb{R}^{3}$ with the following properties:

$$
\begin{gathered}
\mathbf{v} \in \bigcap_{k=0}^{4} W^{k, \infty}\left(\left[0, T^{\prime}\right] ; H^{4-k}(\Omega)\right) \\
\|\mathbf{v}\|_{0,4}+\|\mathbf{v}\|_{1,3}+\|\mathbf{v}\|_{2,2}+\|\mathbf{v}\|_{3,1}+\|\mathbf{v}\|_{4,0} \leq M \\
\operatorname{div} \mathbf{v}=0 \\
\left.\mathbf{v}\right|_{\partial \Omega}=\mathbf{0} \\
\mathbf{v}(\mathbf{x}, 0)=\mathbf{v}_{0}(\mathbf{x}), \frac{\partial \mathbf{v}}{\partial t}(\mathbf{x}, 0)=\mathbf{v}_{1}(\mathbf{x}), \frac{\partial^{2} \mathbf{v}}{\partial t^{2}}(\mathbf{x}, 0)=\mathbf{v}_{2}(\mathbf{x}), \frac{\partial^{3} \mathbf{v}}{\partial t^{3}}(\mathbf{x}, 0)=\mathbf{v}_{3}(\mathbf{x}) .
\end{gathered}
$$

Here $\|\cdot\|_{k, l}$ denotes the norm in $W^{k, \infty}\left(\left[0, T^{\prime}\right] ; H^{l}(\Omega)\right)$. In $Z\left(M, T^{\prime}\right)$, we introduce the metric

$$
d(\mathbf{v}, \mathbf{w})=\|\mathbf{v}-\mathbf{w}\|_{0,3}+\|\mathbf{v}-\mathbf{w}\|_{1,2}+\|\mathbf{v}-\mathbf{w}\|_{2,1}+\|\mathbf{v}-\mathbf{w}\|_{3,0} .
$$

It is easy to see that $Z\left(M, T^{\prime}\right)$ with this metric is complete. We refer to [10] for a proof that it is not empty (provided $M$ is large enough).

We note that equation (13) is of the same type as the problem (16) considered in [10]. The quantity

$$
\int\left(\gamma\left(|\mathbf{R}|^{2}\right) R_{j} R_{l} \delta_{i k}+2 \gamma^{\prime}\left(|\mathbf{R}|^{2}\right) R_{i} R_{j} R_{k} R_{l}\right) \psi^{m} d \mathbf{R}
$$

assumes the role of $C_{i j k l}$ in [10]. We note that the symmetry condition

$$
C_{i j k l}=C_{k l i j}
$$

and the strong ellipticity condition

$$
C_{i j k l} \zeta_{i} \zeta_{k} \eta_{j} \eta_{l} \geq \kappa|\zeta|^{2}|\eta|^{2}, \forall \zeta, \eta \in \mathbb{R}^{3}
$$

with $\kappa>0$ depending continuously on $\psi \in\left\{\psi \in L^{1} \mid \psi \geq 0, \int \psi d \mathbf{R}=1\right\}$, are satisfied, because we have assumed $\gamma$ to be an increasing function of $|\mathbf{R}|^{2}$. Hence the results of [10] are applicable to equation (13), and most of the proof in the following Section will be concerned with solving the diffusion equation (12). 


\section{Proof of the theorem}

In order to show that $\Sigma$ is a contraction in $Z\left(M, T^{\prime}\right)$, we shall have to choose $M$ sufficiently large and $T^{\prime}$ sufficiently small. The estimates used in the proof will involve bounds of the form $K\left(M, T^{\prime}, a_{1}, a_{2}, ..\right)$, where the size of $a_{1}, a_{2}$ etc. can be kept within given bounds. It is important that the size of $K$ for large $M$ can be controlled by choosing $T^{\prime}$ small enough. This leads us to the following definition.

\section{Definition:}

$A$ continuous function $K\left(M, T^{\prime}, a_{1}, a_{2}, \ldots\right): \mathbb{R}^{+} \times \mathbb{R}^{+} \times \mathbb{R}^{+} \ldots \rightarrow \mathbb{R}^{+}$is called controllable, if there are continuous positively valued functions $\tau\left(M, a_{1}, a_{2}, \ldots\right)$ and $\omega\left(a_{1}, a_{2}, \ldots\right)$ such that $K\left(M, T^{\prime}, a_{1}, a_{2}, \ldots\right) \leq \omega\left(a_{1}, a_{2}, \ldots\right)$ as long as $T^{\prime} \leq \tau\left(M, a_{1}, a_{2}, \ldots\right)$.

We are concerned with the solution of equations of the form (12), i.e. for given $\mathbf{v} \in Z\left(M, T^{\prime}\right)$, we have to find $\psi$ satisfying

$$
\begin{gathered}
\left(\frac{\partial}{\partial t}+\left(\mathbf{v} \cdot \nabla_{\mathbf{x}}\right)\right) \psi= \\
\alpha \Delta_{\mathbf{R}} \psi+\operatorname{div}_{\mathbf{R}}\left(-\left(\nabla_{\mathbf{x}} \mathbf{v}\right) \cdot \mathbf{R} \psi-\beta \mathbf{F}(\mathbf{R}) \psi\right) \\
\psi(\mathbf{R}, \mathbf{x}, 0)=\psi_{0}(\mathbf{R}, \mathbf{x}) .
\end{gathered}
$$

The following lemma holds:

\section{Lemma 1:}

Given $\mathbf{v} \in Z\left(M, T^{\prime}\right)$, there exists a unique solution of (22) which has the regularity

$$
\psi \in \bigcap_{k=0}^{3} \bigcap_{l=0}^{3-k} C^{k}\left(\left[0, T^{\prime}\right] ; H^{l}\left(\Omega ; X_{8-2 k-2 l}\right)\right) .
$$

Let $\|\cdot\|_{(n)}$ be the norm in

$$
\bigcap_{k=0}^{3} \bigcap_{l=0}^{3-k} C^{k}\left(\left[0, T^{\prime}\right] ; H^{l}\left(\Omega ; X_{n, 8-2 k-2 l}\right)\right)
$$

For every $n$, we have an estimate of the form

$$
\|\psi\|_{(n)} \leq C_{n} \cdot K\left(M, T^{\prime}, n\right)
$$

where $C_{n}$ depends only on the initial data and $K$ is controllable.

We note that it is in general neither possible nor necessary to obtain a bound for $K\left(M, T^{\prime}, n\right)$ which is independent of $n$. By using (23) and equation (22), we find, moreover, that

$$
\frac{\partial \psi}{\partial t}+\left(\mathbf{v} \cdot \nabla_{\mathbf{x}}\right) \psi \in \bigcap_{k=0}^{3} \bigcap_{l=0}^{3-k} W^{k, \infty}\left(\left[0, T^{\prime}\right] ; H^{l}\left(\Omega ; X_{6-2 k-2 l}\right)\right) .
$$


In addition to (22), let us consider a second equation of the same form;

$$
\begin{gathered}
\left(\frac{\partial}{\partial t}+\left(\tilde{\mathbf{v}} \cdot \nabla_{\mathbf{x}}\right)\right) \tilde{\psi}= \\
\alpha \Delta_{\mathbf{R}} \tilde{\psi}+\operatorname{div}_{\mathbf{R}}\left(\left(-\nabla_{\mathbf{x}} \tilde{\mathbf{v}}\right) \cdot \mathbf{R} \tilde{\psi}-\beta \mathbf{F}(\mathbf{R}) \tilde{\psi}\right) \\
\tilde{\psi}(\mathbf{R}, \mathbf{x}, 0)=\psi_{0}(\mathbf{R}, \mathbf{x})
\end{gathered}
$$

The following result holds.

\section{Lemma 2:}

Let $\mathbf{v}, \tilde{\mathbf{v}} \in Z\left(M, T^{\prime}\right)$ be given. Let $\|\cdot\|_{[n]}$ denote the norm in

$$
\bigcap_{k=0}^{2} \bigcap_{l=0}^{2-k} C^{k}\left(\left[0, T^{\prime}\right] ; H^{l}\left(\Omega ; X_{6-2 k-2 l}\right)\right) .
$$

Then for every $n$, we have an estimate of the form

$$
\|\psi-\tilde{\psi}\|_{[n]} \leq K\left(M, T^{\prime}, n\right) d(\mathbf{v}, \tilde{\mathbf{v}}) .
$$

Here $d(\mathbf{v}, \tilde{\mathbf{v}})$ is as defined in (18). The function $K\left(M, T^{\prime}, n\right)$ tends to zero as $T^{\prime} \rightarrow 0$.

Using the equations, we find, as a consequence, a similar estimate for

$$
\left(\frac{\partial}{\partial t}+\left(\mathbf{v} \cdot \nabla_{\mathbf{x}}\right)\right) \psi-\left(\frac{\partial}{\partial t}+\left(\tilde{\mathbf{v}} \cdot \nabla_{\mathbf{x}}\right)\right) \tilde{\psi}
$$

in the space

$$
\bigcap_{k=0}^{2} \bigcap_{l=0}^{2-k} C^{k}\left(\left[0, T^{\prime}\right] ; H^{l}\left(\Omega ; X_{4-2 k-2 l}\right)\right) .
$$

We can apply Lemmas 3 and 4 of [10] to the solution of equation (13). By combining the resulting estimates with Lemmas 1 and 2 above, it follows easily that $\Sigma$ is a contraction in $Z\left(M, T^{\prime}\right)$ if $M$ is chosen sufficiently large and $T^{\prime}$ is chosen sufficiently small. The theorem follows immediately. The rest of the paper will therefore be concerned with the proofs of Lemmas 1 and 2.

We introduce Lagrangian coordinates, which we shall denote by $\mathbf{X}$. Let $\mathbf{x}(\mathbf{X}, t)$ denote the Eulerian coordinate corresponding to $\mathbf{X}$, i.e. the solution of the problem

$$
\frac{\partial}{\partial t} \mathbf{x}(\mathbf{X}, t)=\mathbf{v}(\mathbf{x}(\mathbf{X}, t), t), \mathbf{x}(\mathbf{X}, 0)=\mathbf{X} .
$$

Let $\phi(\mathbf{R}, \mathbf{X}, t)=\psi(\mathbf{R}, \mathbf{x}(\mathbf{X}, t), t)$. Equation (22) can then be written in the alternative form

$$
\begin{gathered}
\frac{\partial}{\partial t} \phi(\mathbf{R}, \mathbf{X}, t)=\alpha \Delta_{\mathbf{R}} \phi+\operatorname{div}_{\mathbf{R}}\left(-\left(\nabla_{\mathbf{x}} \mathbf{v}(\mathbf{x}(\mathbf{X}, t), t)\right) \cdot \mathbf{R} \phi-\beta \mathbf{F}(\mathbf{R}) \phi\right), \\
\phi(\mathbf{R}, \mathbf{X}, 0)=\psi_{0}(\mathbf{R}, \mathbf{X}) .
\end{gathered}
$$


This is a parabolic equation for $\phi$, in which the only derivatives are with respect to $\mathbf{R}$ and $t$, while $\mathbf{X}$ only appears as a parameter in the coefficients and initial conditions. We note that the transformation $(\mathbf{X}, t) \rightarrow(\mathbf{x}(\mathbf{X}, t), t)$ is smooth enough so that if $\psi$ has the regularity claimed by Lemma 1 , then so does $\phi$ and vice versa.

The fact that solutions of (33) with the regularity claimed by Lemma 1 are unique follows from a straightforward application of the maximum principle. It also follows from the maximum principle that positivity is preserved, and by integrating both sides of (33) we find that $\int \phi(\mathbf{R}, \mathbf{X}, t) d \mathbf{R}=\int \psi(\mathbf{R}, \mathbf{x}, t) d \mathbf{R}=1$ for all $t$. To show the existence of solutions, we cannot use standard results available in the literature because the coefficients of the equation are unbounded. To get around this difficulty, we use a sequence of approximating problems with bounded coefficients, for which we derive uniform estimates. The cases of infinitely, and respectively, finitely extensible dumbbells have to be treated somewhat differently, and we shall first deal with infinitely extensible dumbbells.

Let $\chi(\mathbf{R})$ be a $C^{\infty}$-function such that $\chi(0)=1, \chi$ is a monotone decreasing function of $|\mathbf{R}|$, and $\chi(\mathbf{R})=|\mathbf{R}|^{-\nu}$ for large $|\mathbf{R}|$, where $\nu$ is a sufficiently large number. For $N \in \mathbb{N}$, let $\chi_{N}(\mathbf{R})=\chi(\mathbf{R} / N)$. We now replace (33) by the approximate problem

$$
\begin{aligned}
& \frac{\partial \phi_{N}}{\partial t}=\alpha \Delta_{\mathbf{R}} \phi_{N}+\operatorname{div}_{\mathbf{R}}[\left.\chi_{N}(\mathbf{R})\left(-\left(\nabla_{\boldsymbol{x}} \mathbf{v}(\mathbf{x}(\mathbf{X}, t), t)\right) \cdot \mathbf{R} \phi_{N}-\beta \mathbf{F}(\mathbf{R}) \phi_{N}\right)\right], \\
& \phi_{N}(\mathbf{R}, \mathbf{X}, 0)=\psi_{0}(\mathbf{R}, \mathbf{X}) .
\end{aligned}
$$

We note that equation (34) has bounded coefficients. Hence the existence of solutions and their decay as $|\mathbf{R}| \rightarrow \infty$ are easily established. We now multiply (34) by $|\mathbf{R}|^{2 n}$ and integrate. This yields after an integration by parts

$$
\begin{gathered}
\frac{\partial}{\partial t} \int|\mathbf{R}|^{2 n} \phi_{N}(\mathbf{R}, \mathbf{X}, t) d \mathbf{R}=\alpha\left(4 n^{2}+2 n\right) \int|\mathbf{R}|^{2 n-2} \phi_{N}(\mathbf{R}, \mathbf{X}, t) d \mathbf{R} \\
+2 n \int|\mathbf{R}|^{2 n-2} \chi_{N}(\mathbf{R})\left[\mathbf{R} \cdot\left(\nabla_{\mathbf{x}} \mathbf{v}(\mathbf{x}(\mathbf{X}, t), t)\right) \cdot \mathbf{R}+\beta \mathbf{R} \cdot \mathbf{F}(\mathbf{R})\right] \phi_{N}(\mathbf{R}, \mathbf{X}, t) d \mathbf{R} .
\end{gathered}
$$

The term $\mathbf{R} \cdot \mathbf{F}(\mathbf{R})$ is negative, and all other terms on the right hand side of (35) can be estimated by a constant (depending on $n$ but not on $N$ ) times $\int\left(1+|\mathbf{R}|^{2 n}\right) \phi_{N}(\mathbf{R}, \mathbf{X}, t) d \mathbf{R}$. Hence (35) yields a bound of the form

$$
\int|\mathbf{R}|^{2 n} \phi_{N}(\mathbf{R}, \mathbf{X}, t) \leq K(n, M t) \int\left(1+|\mathbf{R}|^{2 n}\right) \psi_{0}(\mathbf{R}, \mathbf{X}) d \mathbf{R} .
$$

We next derive estimates for derivatives with respect to $\mathbf{R}$. We differentiate (34) with respect to $R_{i}$ and obtain

$$
\begin{aligned}
\frac{\partial^{2} \phi_{N}}{\partial R_{i} \partial t} & =\alpha \Delta_{\mathbf{R}} \frac{\partial \phi_{N}}{\partial R_{i}}+\operatorname{div}_{\mathbf{R}}\left[\chi_{N}(\mathbf{R})\left(-\left(\nabla_{\mathbf{x}} \mathbf{v}(\mathbf{x}(\mathbf{X}, t), t)\right) \cdot \mathbf{R}-\beta \mathbf{F}(\mathbf{R})\right) \frac{\partial \phi_{N}}{\partial R_{i}}\right] \\
& +\operatorname{div}_{\mathbf{R}}\left[\frac{\partial}{\partial R_{i}}\left(\chi_{N}(\mathbf{R})\left(-\left(\nabla_{\mathbf{x}} \mathbf{v}(\mathbf{x}(\mathbf{X}, t), t)\right) \cdot \mathbf{R}-\beta \mathbf{F}(\mathbf{R})\right)\right) \phi_{N}\right]
\end{aligned}
$$




$$
\frac{\partial \phi_{N}}{\partial R_{i}}(\mathbf{R}, \mathbf{X}, 0)=\frac{\partial \psi_{0}}{\partial R_{i}}(\mathbf{R}, \mathbf{X})
$$

The last term on the right hand side of this equation can be further transformed as follows:

$$
\begin{gathered}
\operatorname{div}_{\mathbf{R}}\left[\frac{\partial}{\partial R_{i}}\left(\chi_{N}(\mathbf{R})\left(-\left(\nabla_{\mathbf{x}} \mathbf{v}(\mathbf{x}(\mathbf{X}, t), t)\right) \cdot \mathbf{R}-\beta \mathbf{F}(\mathbf{R})\right)\right) \phi_{N}\right] \\
=\phi_{N} \operatorname{div}_{\mathbf{R}}\left[\frac{\partial}{\partial R_{i}}\left(\chi_{N}(\mathbf{R})\left(-\left(\nabla_{x} \mathbf{v}\right) \cdot \mathbf{R}-\beta \mathbf{F}(\mathbf{R})\right)\right)\right] \\
+\frac{\partial \phi_{N}}{\partial R_{j}}\left[\frac{\partial \chi_{N}}{\partial R_{i}}\left(-\frac{\partial v_{j}}{\partial x_{k}} R_{k}-\beta F_{j}(\mathbf{R})\right)+\chi_{N}(\mathbf{R})\left(-\frac{\partial v_{j}}{\partial x_{i}}-\beta \frac{\partial F_{j}}{\partial R_{i}}\right)\right] .
\end{gathered}
$$

Since $\partial \phi_{N} / \partial R_{i}$ is not positive, we cannot proceed in exact analogy to equation (35) above. Instead, we first decompose $\partial \phi_{N} / \partial R_{i}=\pi_{i}^{+}-\pi_{i}^{-}$, where $\pi_{i}^{+}$and $\pi_{i}^{-}$are solutions to the problems,

$$
\begin{gathered}
\frac{\partial \pi_{i}^{+}}{\partial t}=\alpha \Delta_{\mathbf{R}} \pi_{i}^{+}+\operatorname{div}_{\mathbf{R}}\left[\chi_{N}(\mathbf{R})\left(-\left(\nabla_{\mathbf{x}} \mathbf{v}\right) \cdot \mathbf{R} \pi_{i}^{+}-\beta \mathbf{F}(\mathbf{R}) \pi_{i}^{+}\right)\right]+s_{i}^{+}, \\
\pi_{i}^{+}(\mathbf{R}, \mathbf{X}, 0)=\max \left(\frac{\partial \psi_{0}}{\partial R_{i}}(\mathbf{R}, \mathbf{X}), 0\right),
\end{gathered}
$$

and, respectively,

$$
\begin{gathered}
\frac{\partial \pi_{i}^{-}}{\partial t}=\alpha \Delta_{\mathbf{R}} \pi_{i}^{-}+\operatorname{div}_{\mathbf{R}}\left[\chi_{N}(\mathbf{R})\left(-\left(\nabla_{\mathbf{x}} \mathbf{v}\right) \cdot \mathbf{R} \pi_{i}^{-}-\beta \mathbf{F}(\mathbf{R}) \pi_{i}^{-}\right)\right]-s_{i}^{-}, \\
\pi_{i}^{-}(\mathbf{R}, \mathbf{X}, 0)=\max \left(-\frac{\partial \psi_{0}}{\partial R_{i}}(\mathbf{R}, \mathbf{X}), 0\right) .
\end{gathered}
$$

Here $s_{i}^{+}$and $s_{i}^{-}$denote the positive and negative parts of the expression in (38). Since $\pi_{i}^{+}$ and $\pi_{i}^{-}$are positive, we can now proceed as above. In place of (35), we obtain

$$
\begin{gathered}
\frac{\partial}{\partial t} \int|\mathbf{R}|^{2 n} \pi_{i}^{+}(\mathbf{R}, \mathbf{X}, t) d \mathbf{R}=\alpha\left(4 n^{2}+2 n\right) \int|\mathbf{R}|^{2 n-2} \pi_{i}^{+}(\mathbf{R}, \mathbf{X}, t) d \mathbf{R} \\
+2 n \int|\mathbf{R}|^{2 n-2} \chi_{N}(\mathbf{R})\left[\mathbf{R} \cdot\left(\nabla_{\mathbf{x}} \mathbf{v}(\mathbf{x}(\mathbf{X}, t), t)\right) \cdot \mathbf{R}+\beta \mathbf{R} \cdot \mathbf{F}(\mathbf{R})\right] \pi_{i}^{+}(\mathbf{R}, \mathbf{X}, t) d \mathbf{R}+\int|\mathbf{R}|^{2 n} s_{i}^{+} d \mathbf{R},
\end{gathered}
$$

and an analogous equation for $\pi_{i}^{-}$. (A minor problem in deriving (41) arises from the fact that $\Delta \pi_{i}^{+}$does not necessarily exist at $t=0$, but we may justify the argument by approximating the initial value of $\pi_{i}^{+}$by a sequence of smoother functions and then passing to the limit.) Using (38), we find that

$$
\int|\mathbf{R}|^{2 n} s_{i}^{+} d \mathbf{R} \leq \int|\mathbf{R}|^{2 n} \phi_{N}\left|\operatorname{div}_{\mathbf{R}}\left[\frac{\partial}{\partial R_{i}}\left(\chi_{N}(\mathbf{R})\left(-\left(\nabla_{x} \mathbf{v}\right) \cdot \mathbf{R}-\beta \mathbf{F}(\mathbf{R})\right)\right)\right]\right| d \mathbf{R}
$$




$$
+\int|\mathbf{R}|^{2 n}\left(\pi_{j}^{+}+\pi_{j}^{-}\right)\left[\left|\frac{\partial \chi_{N}}{\partial R_{i}}\right|\left(\left|\frac{\partial v_{j}}{\partial x_{k}} R_{k}\right|+\beta\left|F_{j}(\mathbf{R})\right|\right)+\chi_{N}(\mathbf{R})\left(\left|\frac{\partial v_{j}}{\partial x_{i}}\right|+\beta\left|\frac{\partial F_{j}}{\partial R_{i}}\right|\right)\right] d \mathbf{R} .
$$

The first integral on the right hand side of (42) involves only $\phi_{N}$ and no derivatives of $\phi_{N}$; it can hence be estimated using (36) above. The integrand in the second integral can be estimated for large $|\mathbf{R}|$ by a constant times $\chi_{N}(\mathbf{R})\left(M|\mathbf{R}|^{2 n}+|\mathbf{R}|^{2 n-1}|\mathbf{F}(\mathbf{R})|\right) \sum_{j}\left(\pi_{j}^{+}+\pi_{j}^{-}\right)$. From (41), we therefore obtain an estimate of the form

$$
\begin{gathered}
\frac{\partial}{\partial t} \int|\mathbf{R}|^{2 n} \pi_{i}^{+}(\mathbf{R}, \mathbf{X}, t) d \mathbf{R} \leq K_{1}(n, M) \int\left(1+|\mathbf{R}|^{2 n}\right) \pi_{i}^{+}(\mathbf{R}, \mathbf{X}, t) d \mathbf{R}+K_{2}(n, M) \\
+2 n \int|\mathbf{R}|^{2 n-2} \chi_{N}(\mathbf{R}) \beta \mathbf{R} \cdot \mathbf{F}(\mathbf{R}) \pi_{i}^{+}(\mathbf{R}, \mathbf{X}, t) d \mathbf{R} \\
+K_{3} \int\left(1+|\mathbf{R}|^{2 n-1} \chi_{N}(\mathbf{R})|\mathbf{F}(\mathbf{R})|+M_{N}(\mathbf{R})|\mathbf{R}|^{2 n}\right) \sum_{j}\left(\pi_{j}^{+}+\pi_{j}^{-}\right)(\mathbf{R}, \mathbf{X}, t) d \mathbf{R}
\end{gathered}
$$

Note that $K_{3}$ does not depend on $n$, but that the previous term in (43), which is negative, has a factor $n$ in front. By summing the inequalities corresponding to (43) over all values of $i$ and all choices of + or - , we can therefore get bounds for $\int|\mathbf{R}|^{2 n} \pi_{i}^{+} d \mathbf{R}$. As a consequence, we have found estimates for the first derivatives of $\phi_{N}$. Bounds for higher derivatives are obtained in an analogous fashion after differentiating (34) with respect to the components of $\mathbf{R}$. The calculation involves higher derivatives of $\phi_{N}$ than are known to exist at $t=0$, but the argument can be justified by approximating the initial datum by smoother functions and passing to the limit. Proceeding in this fashion, we find that

$$
\phi_{N} \in L^{\infty}\left(\left[0, T^{\prime}\right] ; L^{2}\left(\Omega ; X_{8}\right)\right) .
$$

Derivatives of $\phi_{N}$ with respect to $\mathbf{X}$ can be estimated by taking difference quotients in (34), estimating the difference quotients of $\phi_{N}$ and then taking limits. Problems near the boundary of $\Omega$ are avoided by first extending $\mathbf{v}$ and $\psi_{0}$ so that they are defined for $\mathbf{X}$ outside $\Omega$. This can be done in such a fashion that the extended functions have the same level of regularity as the original ones. Mixed derivatives with respect to $\mathbf{R}$ and $\mathbf{X}$ can be estimated by taking difference quotients with respect to $\mathbf{X}$ after first differentiating with respect to $\mathbf{R}$. The calculations are tedious but straightforward and we omit them. We find in this way that

$$
\phi_{N} \in \bigcap_{l=0}^{3} L^{\infty}\left(\left[0, T^{\prime}\right] ; H^{l}\left(\Omega ; X_{8-2 l}\right)\right) .
$$

Finally, temporal derivatives of $\phi_{N}$ can be estimated by using the equation itself. This yields

$$
\phi_{N} \in \bigcap_{k=0}^{3} \bigcap_{l=0}^{3-k} W^{k, \infty}\left(\left[0, T^{\prime}\right] ; H^{l}\left(\Omega ; X_{8-2 k-2 l}\right)\right) .
$$

All these estimates are uniform in $N$. 
Using the uniform bounds on the $\phi_{N}$, we can extract a subsequence which converges, in the sense of distributions, to a solution $\phi$ of (33). Unfortunately, it is not a priori clear that

$$
\phi \in \bigcap_{k=0}^{3} \bigcap_{l=0}^{3-k} W^{k, \infty}\left(\left[0, T^{\prime}\right] ; H^{l}\left(\Omega ; X_{8-2 k-2 l}\right)\right) .
$$

To show this, we need a separate argument. We approximate the initial datum $\psi_{0}$ by a sequence $\psi_{0}^{M}$ such that $\psi_{0}^{M}$ has the additional regularity

$$
\psi_{0}^{M} \in \bigcap_{k=0}^{4} H^{k}\left(\Omega ; X_{10-2 k}\right),
$$

and $\psi_{0}^{M} \rightarrow \psi_{0}$ in the topology of

$$
\bigcap_{k=0}^{4} H^{k}\left(\Omega ; X_{8-2 k}\right) .
$$

Let $\phi_{N}^{M}$ be the solution of (34) which is obtained when the initial datum is replaced by $\psi_{0}^{M}$. Then the same argument as above yields

$$
\phi_{N}^{M} \in \bigcap_{k=0}^{3} \bigcap_{l=0}^{3-k} W^{k, \infty}\left(\left[0, T^{\prime}\right] ; H^{l}\left(\Omega ; X_{10-2 k-2 l}\right)\right) ;
$$

moreover, in the topology of

$$
\bigcap_{k=0}^{3} \bigcap_{l=0}^{3-k} W^{k, \infty}\left(\left[0, T^{\prime}\right] ; H^{l}\left(\Omega ; X_{8-2 k-2 l}\right)\right),
$$

we have $\phi_{N}^{M} \rightarrow \phi_{N}$ uniformly in $N$. From (50) and (34), we conclude that

$$
\frac{\partial \phi_{N}^{M}}{\partial t} \in \bigcap_{k=0}^{3} \bigcap_{l=0}^{3-k} W^{k, \infty}\left(\left[0, T^{\prime}\right] ; H^{l}\left(\Omega ; X_{8-2 k-2 l}\right)\right) .
$$

From (50) and (52) it follows that

$$
\phi_{N}^{M} \in \bigcap_{k=0}^{3} \bigcap_{l=0}^{3-k} C^{k}\left(\left[0, T^{\prime}\right] ; H^{l}\left(\Omega ; X_{8-2 k-2 l}\right)\right) .
$$

For fixed $M$, the bounds for $\phi_{N}^{M}$ are again uniform in $N$. Let now $\Pi_{n}$ be a reflexive Banach space such that

$$
\left[\bigcap_{k=0}^{3} \bigcap_{l=0}^{3-k} W^{k, \infty}\left(\left[0, T^{\prime}\right] ; H^{l}\left(\Omega ; X_{n, 10-2 k-2 l}\right)\right)\right] \cap\left[\bigcap_{k=0}^{3} \bigcap_{l=0}^{3-k} W^{k+1, \infty}\left(\left[0, T^{\prime}\right] ; H^{l}\left(\Omega ; X_{n, 8-2 k-2 l}\right)\right)\right]
$$




$$
\subset \Pi_{n} \subset \bigcap_{k=0}^{3} \bigcap_{l=0}^{3-k} C^{k}\left(\left[0, T^{\prime}\right] ; H^{l}\left(\Omega ; X_{n-1,8-2 k-2 l}\right)\right)
$$

Such a space $\Pi_{n}$ can be constructed using appropriate Sobolev norms. Let $\Pi=\bigcap_{n \in \mathbb{N}} \Pi_{n}$. Then, for every $M$, we have a uniform bound for $\phi_{N}^{M}$ in the topology of $\Pi$, hence we can extract a subsequence which converges distributionally to an element $\phi^{M}$ of $\Pi$. Since, for $M \rightarrow \infty, \phi_{N}^{M}$ converges to $\phi_{N}$ uniformly in $N$, we find that the sequence $\phi^{M}$ also converges in

$$
\bigcap_{k=0}^{3} \bigcap_{l=0}^{3-k} C^{k}\left(\left[0, T^{\prime}\right] ; H^{l}\left(\Omega ; X_{8-2 k-2 l}\right)\right)
$$

By taking $\phi=\lim _{M \rightarrow \infty} \phi^{M}$, we obtain a solution with the desired regularity. This concludes the proof of Lemma 1 for the case of the infinitely extensible dumbbell.

The proof of Lemma 2 is based on the same type of estimates, applied to the function $\psi-\tilde{\psi}$. We omit the details.

For the case of finitely extensible dumbbells, we use approximation by infinitely extensible dumbbells. We extend the initial datum $\psi_{0}$ by zero for $|\mathbf{R}|>R_{0}$, and we define an approximation $\gamma_{\epsilon}$ to the spring constant $\gamma$ as follows. Let $\chi$ be a $C^{\infty}$-function $[0,1] \rightarrow[0,1]$ such that $\chi(s)=1$ in a neighborhood of 0 and $\chi(s)=0$ in a neighborhood of 1 . Let $\chi_{\epsilon}(\mathbf{R})=\chi\left(\frac{2}{\epsilon}\left(|\mathbf{R}|-R_{0}+\epsilon\right)\right)$. Then we define

$$
\gamma_{\epsilon}\left(|\mathbf{R}|^{2}\right)= \begin{cases}\gamma\left(|\mathbf{R}|^{2}\right), & \text { if }|\mathbf{R}| \leq R_{0}-\epsilon, \\ \gamma\left(\left(R_{0}-\frac{\epsilon}{2}\right)^{2}\right), & \text { if }|\mathbf{R}| \geq R_{0}-\frac{\epsilon}{2}, \\ \chi_{\epsilon}(\mathbf{R}) \gamma\left(|\mathbf{R}|^{2}\right)+\left(1-\chi_{\epsilon}(\mathbf{R})\right) \gamma\left(\left(R_{0}-\frac{\epsilon}{2}\right)^{2}\right), & \text { if } R_{0}-\epsilon<|\mathbf{R}|<R_{0}-\frac{\epsilon}{2}\end{cases}
$$

We now consider the problem with spring constant $\gamma_{\epsilon}$, which is infinitely extensible and satisfies the assumptions which we required for the infinitely extensible case. We now define

$$
w_{\epsilon}(\mathbf{R})= \begin{cases}\frac{1}{R_{0}^{2}-|\mathbf{R}|^{2}}, & \text { if }|\mathbf{R}| \leq R_{0}-\epsilon \\ \frac{1}{\epsilon\left(2 R_{0}-\epsilon\right)}+\frac{2\left(R_{0}-\epsilon\right)}{\epsilon^{2}\left(2 R_{0}-\epsilon\right)^{2}}\left(|\mathbf{R}|-R_{0}+\epsilon\right), & \text { if }|\mathbf{R}|>R_{0}-\epsilon\end{cases}
$$

The function $w_{\epsilon}$ and its gradient are continuous at $|\mathbf{R}|=R_{0}$, and $w_{\epsilon}$ has linear growth at infinity. We now repeat the estimates above, but instead of multiplying the equation by powers of $|\mathbf{R}|$, we multiply by powers of $w_{\epsilon}(|\mathbf{R}|)$. The resulting estimates turn out to be uniform in $\epsilon$ as $\epsilon \rightarrow 0$, and Lemma 1 follows by passing to the limit. 


\section{References}

[1] R.B. Bird, O. Hassager, R.C. Armstrong and C.F. Curtiss, Dynamics of Polymeric Liquids, Vol. 2: Kinetic Theory, Wiley 1977 and 1987

[2] C. Chen and W. von Wahl, Das Rand-Anfangswertproblem für quasilineare Wellengleichungen in Sobolevräumen niedriger Ordnung, J. reine angew. Math. 337 (1982), $77-112$

[3] C.M. Dafermos and W.J. Hrusa, Energy methods for quasilinear hyperbolic initialboundary value problems. Applications to elastodynamics, Arch. Rat. Mech. Anal. 87 (1985), 267-292

[4] E.G. Ebin and R.A. Saxton, The initial value problem for elastodynamics of incompressible bodies, Arch. Rat. Mech. Anal. 94 (1986), 15-38

[5] W.J. Hrusa and M. Renardy, An existence theorem for the Dirichlet problem in the elastodynamics of incompressible materials, Arch. Rat. Mech. Anal. 102 (1988), 95-117

[6] T.J.R. Hughes, T. Kato and J.E. Marsden, Well-posed quasi-linear second-order hyperbolic systems with applications to nonlinear elastodynamics and general relativity, Arch. Rat. Mech. Anal. 63 (1976), 273-284

[7] T. Kato, Linear and quasi-linear equations of hyperbolic type, in: G. da Prato and G. Geymonat (eds.), Hyperbolicity, Centro Internazionale Matematico Estivo, II ciclo, Cortona 1976, 125-191

[8] M. Renardy, W.J. Hrusa and J.A. Nohel, Mathematical Problems in Viscoelasticity, Longman 1987

[9] M. Renardy, Local existence theorems for the first and second initial-boundary value problems for a weakly non-Newtonian fluid, Arch. Rat. Mech. Anal. 83 (1983), pp. 229-244

[10] M. Renardy, Local existence of solutions of the Dirichlet initial-boundary value problem for incompressible hypoelastic materials, submitted to SIAM J. Math. Anal.

[11] S. Schochet, The incompressible limit in nonlinear elasticity, Comm. Math. Phys. 102 (1985), 207-215 
Daniel D. Joseph, Generalization of the Foscolo-Gibilaro Analysis of Dynamic Waves

A. Narain and D.D. Joseph, Note on the Balance of Energy at a Phase Change Interface

Daniel D. Joseph, Remarks on inertial radii, persistent normal stresses, secondary motions, and non-elastic extensional viscosities

D. D. Joseph, Mathematical Problems Associated with the Elasticity of Liquids

Henry C. Simpson and Scott J. Spector, Some Necessary Conditions at an Internal Boundary for Minimizers in Finite Elasticity

Peter Gritzmann and Victor Klee, On the 0-1 Maximization of Positive Definite Quadratic Forms

Fu-Cho Pu and D.H. Sattinger, The Yang-Baxter Equations and Differential Identities

Avner Friedman and Fernando Reitich, A Hyperbolic Inverse Problem Arising in the Evolution of Combustion Aerosol

E.G. Kalnins, Raphael D. Levine and Willard Miller, Jr., Conformal Symmetries and Generalized Recurrences for Heat and Schrödinger Equations in One Spatial Dimension

Wang Jinghua and Gerald Warnecke, On Entropy Consistency of Large Time Step Godunov and Glimm Schemes

C. Guillopé and J.C. Saut, Existence Results for the Flow of Viscoelastic Fluids with a Differential Constitutive Law

H.L. Bodlaender, P. Gritzmann, V. Klee and J. Van Leeuwen Computational Complexity of Norm-Maximization

Li Ta-tsien (Li Da-qian) and Yu Xin, Life-Span of Classical Solutions to Fully Nonlinear Wave Equations

Jong-Shenq Guo, A Variational Inequality Associated with a Lubrication Problem

Jong-Shenq Guo, On the Semilinear Elliptic Equation $\Delta w-\frac{1}{2} y \cdot \nabla w+\lambda w-w^{-\beta}=0$ in $R^{n}$

Andrew E. Yagle, Inversion of the Bloch transform in magnetic resonance imaging using asymmetric two-component inverse scattering

Bei Hu, A Fiber Tapering Problem

Peter J. Olver, Canonical Variables for BiHamiltonian Systems

Michael Renardy, A Well-Posed Boundary Value Problem for Supercritical Flow of Viscoelastic Fluids of Maxwell Type

Michael Renardy, Ill-Posedness Resulting from Slip As a Possible Explanation of Melt Fracture Michael Renardy, Compatibility Conditions at Corners Between Walls and Inflow Boundaries for Fluids of Maxwell Type

Rolf Rees, The Spectrum of Restricted Resolvable Designs with $r=2$

D. Lewis and J.C. Simo, Nonlinear stability of rotating pseudo-rigid bodies

Robert Hardt and David Kinderlehrer, Variational Principles with Linear Growth

San Yih Lin and Yisong Yang, Computation of Superconductivity in Thin Films

A. Narain, Pressure Driven Flow of Pure Vapor Undergoing Laminar Film Condensation Between Parallel Plates

P.J. Vassiliou, On Local Equivalence for Vector Field Systems

Brian A. Coomes, On Conditions Sufficient for Injectivity of Maps

Yanchun Zhao, A Class of Global Smooth Solutions of the One Dimensional Gas Dynamics System

H. Holden, L. Holden and N.H. Risebro, Some Qualitative Properties of $2 \times 2$ Systems of Conservation Laws of Mixed Type

M. Slemrod, Dynamics of Measured Valued Solutions to a Backward-Forward Heat Equation

Avner Friedman and Jürgen Sprekels, Steady States of Austenitic-Martensitic-Domains in the Ginzburg-Landau Theory of Shape Memory Alloys

Avner Friedman and Bei Hu, Degenerate Hamilton-Jacobi-Bellman Equations in a Bounded Domain

E.G. Kalnins, Willard Miller, Jr., and M.V. Tratnik, Families of Orthogonal and Biorthogonal Polynomials on the N-Sphere

Heinrich Freistühler, On Compact Linear Degeneracy

Matthew Witten, Quantifying the Concepts of Rate and Acceleration/Deceleration of Aging

J.P. Albert and J.L. Bona, Total Positivity and the Stability of Internal Waves in Stratified Fluids of Finite Depth

554 Brian Coomes and Victor Zurkowski, Linearization of Polynomial Flows and Spectra of Derivations 


\section{Recent IMA Preprints (Continued)}

\section{Author/s}

Title

Yuriko Renardy, A Couette-Poiseuille Flow of Two Fluids in a Channel

Michael Renardy, Short wave instabilities resulting from memory slip

Daniel D. Joseph and Michael Renardy, Stokes' first problem for linear viscoelastic fluids with finite memory

Xiaxi Ding, Superlinear Conservation Law with Viscosity

J.L. Ericksen, Liquid Crystals with Variable Degree of Orientation

J.L. Ericksen, Liquid Crystals with Variable Degree of Orientation Waveguide

M.V. Tratnik, Multivariable biorthogonal continuous-discrete Wilson and Racah polynomials

Yisong Yang, Existence of Solutions for a Generalized Yang-Mills Theory

Peter Gritzmann, Laurent Habsieger and Victor Klee, Good and Bad Radii of Convex Polygons Martin Golubitsky, Martin Krupa and Chjan. C. Lim, Time-Reversibility and Particle Sedimentation G. Yin, Recent Progress in Parallel Stochastic Approximations

G. Yin, On H-Valued SA: Finite Dimensional Approximations

Chien-Cheng Chang, Accurate Evaluation of the Effect of Diffusion and Conductivity in Certain Equations

Chien-Cheng Chang and Ruey-Ling Chern, The Effect of Viscous Diffusion in Discrete Vortex Dynamics for Slightly Viscous Flows

Li Ta-Tsien (Li Da-qian) and Zhao Yan-Chun, Global Existence of Classical Solutions to the Typical Free Boundary Problem for General Quasilinear Hyperbolic Systems and its Applications

Thierry Cazenave and Fred B. Weissler, The Structure of Solutions to the Pseudo-Conformally Invariant Nonlinear Schrödinger Equation

Marshall Slemrod and Athanasios E. Tzavaras, A Limiting Viscosity Approach for the Riemann Problem in Isentropic Gas Dynamics

Richard D. James and Scott J. Spector, The Formation of Filamentary Voids in Solids

P.J. Vassiliou, On the Geometry of Semi-Linear Hyperbolic Partial Differential Equations in the Plane Integrable by the Method of Darboux

Jerome V. Moloney and Alan C. Newell, Nonlinear Optics

Keti Tenenblat, A Note on Solutions for the Intrinsic Generalized Wave and Sine-Gordon Equations

P. Szmolyan, Heteroclinic Orbits in Singularly Perturbed Differential Equations

Wenxiong Liu, A Parabolic System Arising In Film Development

Daniel B. Dix, Temporal Asymptotic Behavior of Solutions of the Benjamin-Ono-Burgers Equation

Michael Renardy and Yuriko Renardy, On the nature of boundary conditions for flows with moving free surfaces

Werner A. Stahel, Robust Statistics: From an Intellectual Game to a Consumer Product

Avner Friedman and Fernando Reitich, The Stefan Problem with Small Surface Tension

E.G. Kalnins and W. Miller, Jr., Separation of Variables Methods for Systems of Differential Equations in Mathematical Physics

Mitchell Luskin and George R. Sell, The Construction of Inertial Manifolds for Reaction-Diffusion Equations by Elliptic Regularization

Konstantin Mischaikow, Dynamic Phase Transitions: A Connection Matrix Approach

Philippe Le Floch and Li Tatsien, A Global Asymptotic Expansion for the Solution to the Generalized Riemann Problem

Matthew Witten, Ph.D., Computational Biology: An Overview

Matthew Witten, Ph.D., Peering Inside Living Systems: Physiology in a Supercomputer

Michael Renardy, An existence theorem for model equations resulting from kinetic theories of polymer solutions

Daniel D. Joseph and Luigi Preziosi, Reviews of Modern Physics: Addendum to the Paper "Heat Waves"

Luigi Preziosi, An Invariance Property for the Propagation of Heat and Shear Waves

Gregory M. Constantine and John Bryant, Sequencing of Experiments for Linear and Quadratic Time Effects

Prabir Daripa, On the Computation of the Beltrami Equation in the Complex Plane Philippe Le Floch, Shock Waves for Nonlinear Hyperbolic Systems in Nonconservative Form

A.L. Gorin, D.B. Roe and A.G. Greenberg, On the Complexity of Pattern Recognition Algorithms On a Tree-Structured Parallel Computer

Mark J. Friedman and Eusebius J. Doedel, Numerical computation and continuation of invariant manifolds connecting fixed points 